\title{
Initial Experiences of Laboratory Diagnosis of Coronavirus Disease 2019 (COVID-19) in Singapore General Hospital
}

\section{Dear Editor,}

On 31 December 2019, the World Health Organization (WHO) was alerted to the emergence of a novel zoonotic severe acute respiratory syndrome coronavirus 2 (SARS-CoV-2) in Wuhan, China. ${ }^{1}$ Shortly thereafter, Corman et al published a reverse transcription polymerase chain reaction (PCR) protocol for SARS-CoV-2 on 13 January 2020. ${ }^{2}$ The Department of Molecular Pathology in the Singapore General Hospital put this test into service on 23 January 2020 and was the first to detect the coronavirus disease 2019 (COVID-19) in a patient in Singapore on the same day. The test was clinically validated against the patient's travel history, presenting signs and symptoms and chest radiograph findings. It was also validated against a confirmatory in-house test performed by the National Public Health Laboratory. Although positive controls were provided by the European Virus Archive Global, to avoid any delay in getting the controls shipped to Singapore, a severe acute respiratory syndrome coronavirus extract was used as a positive control since we were aware that the $\mathrm{E}$ gene in both viruses would be detected by the assay.

By 29 February 2020, a total of 3079 tests were performed on 1945 patients; 74 positive results were found in 13 COVID-19 patients. As countries from around the world ramp up tests for COVID-19, we believe our experience is useful for both clinicians and laboratories alike.

For sample collection, a deliberate choice was made to use dry Dacron swabs with no media because these were more likely to be still readily available during a pandemic. When swabs in media were accepted, those with smaller media volumes $(1 \mathrm{~mL})$ resulted in better sensitivity from less sample dilution.

Most of the samples that were received were oropharyngeal swabs. In our experience, swab yields are extremely operator-dependent. For example, a physician was still able to take swabs that were PCR-positive from 2 patients who had 2-4 days of intervening negative PCR after swabs were taken by other physicians. There were also cases when interjacent swabs were negative but were followed by strong positive results, such as in 1 patient who had consumed food and drinks before a negative sample was found. This variability may account for some of the apparent intermittent viral ribonucleic acid (RNA) detection previously reported elsewhere. ${ }^{3}$ In our experience, patients can remain swab-positive for up to 25 days. Hence, adequate training in sample collection is just as critical as having a well-optimised assay. It is also important to collect multiple samples from the same patient.

For RNA extraction, easyMAG (bioMérieux, Marcy-l'Étoile, France) was used because the virus inactivation/lysis step can take place in biosafety cabinets before extraction resumed in automated extractors. It is important to note that not all commonly used lysis buffers are able to effectively inactivate viruses. ${ }^{4}$

To prioritise assay sensitivity, larger extraction volume input $(500 \mu \mathrm{L})$ and lower eluate output $(25 \mu \mathrm{L})$ were chosen, although this had to be balanced against higher PCR inhibition risks. An RNA internal control is therefore important and should be incorporated into the extraction step. This protocol may not work on media other than phosphate-buffered saline.

Initially, the WHO protocol was followed by screening for the $\mathrm{E}$ gene (95\% limit of detection [LOD] 16 copies/reaction) and running a confirmatory test with ORF1ab or RdRp gene assay. However, detection of RdRp is not reliable when the $\mathrm{E}$ gene threshold cycle value is $>30$. We have since switched the confirmatory assay to ORF1b-NSP-14 (95\% LOD 31 copies/reaction) which has much better sensitivity. ${ }^{5}$

Unexpectedly, contaminated primers and probes from reputable manufacturers were encountered repeatedly because they were also producing synthetic E gene oligonucleotides for use in assay validation or as positive controls. This has implications for laboratory-developed tests and rigorous testing of each 
new lot is advisable. All low positive results should be repeated and concurrently with another equally sensitive target such as ORF1b-NSP-14. Laboratories can also select manufacturers-TIB Molbiol GmbH (Berlin, Germany) in our case-that produce only primer probes, not synthetic oligonucleotides.

\section{REFERENCES}

1. World Health Organization. Coronavirus disease (COVID-19) pandemic. Available at: https://www.who.int/emergencies/diseases/ novel-coronavirus-2019. Accessed on 2 March 2020.

2. Corman VM, Landt O, Kaiser M, Molenkamp R, Meijer A, Chu DKW, et al. Detection of 2019 novel coronavirus (2019-nCoV) by real-time RT-PCR. Euro Surveill 2020;25:2000045.

3. Li Y, Yao L, Li J, Chen L, Song Y, Cai Z, et al. Stability issues of RT-PCR testing of SARS-CoV-2 for hospitalized patients clinically diagnosed with COVID-19. J Med Virol 2020;doi:10.1002/ jmv.25786.
4. Smither SJ, Weller SA, Phelps A, Eastaugh L, Ngugi S, O’Brien LM, et al. Buffer AVL alone does not inactivate Ebola virus in a representative clinical sample type. J Clin Microbiol 2015;53: 3148-54.

5. Poon L, Chu D, Peiris M. Detection of 2019 novel coronavirus (2019-nCoV) in suspected human cases by RT-PCR. Available at: https://www.who.int/docs/default-source/coronaviruse peiris-protocol-16-1-20.pdf?sfvrsn=af1aac73_4. Accessed on 2 March 2020.

Kian Sing $\underline{\text { Chan }},{ }^{1} M B B S, F R C P A$, Karrie KK $\underline{\text { Ko }},{ }^{1,2}{ }_{\text {FRCPA, FRCPath, }}$ Tse Hsien Koh, ${ }^{2} M D, P h D$, Danielle WL $\underline{\text { Ho }},{ }^{3} M B B S, M R C P$, Yvonne FZ Chan, ${ }^{3}$ MBChB, MRCP, Thuan Tong Tan, ${ }^{3}{ }_{M R C P}$, PhD, Lynette LE Oon, ${ }^{1} M B B S, F R C P A$

'Department of Molecular Pathology, Singapore General Hospital, Singapore ${ }^{2}$ Department of Microbiology, Singapore General Hospital, Singapore ${ }^{3}$ Department of Infectious Diseases, Singapore General Hospital, Singapore

Address for Correspondence: Dr Koh Tse Hsien, Department of Microbiology, Level 7 Diagnostics Tower, 20 College Road, Academia, Singapore General Hospital, Singapore 169856

Email: koh.tse.hsien@singhealth.com.sg 